\title{
Multi-Parameter Fluctuation Effects on InGaAsP/InP Geiger-Mode Avalanche Photodiodes
}

\author{
Qian Dai ${ }^{1}$, Jie Deng ${ }^{1}$, Zhu Shi ${ }^{1}$, Li-Bo Yu1, and Hai-Zhi Song 1,2,a * \\ 1Southwest Institute of Technical Physics, Section 4-7, Renminnan Road, Chengdu, China \\ ${ }^{2}$ Institute of Fundamental and Frontier Sciences, University of Electronic Science and Technology \\ of China, Section 2-4, Jianshebei Road, Chengdu, Sichuan 610054, China \\ ahzsong1296@163.com
}

Keywords: Optoelectronic device, avalanche photodiode, single photon avalanche diode.

\begin{abstract}
For Geiger-mode avalanche photodiodes, a statistical method is advanced to establish the quantitative correlation between the controllability of structure parameters and the homogeneity of device properties. Setting many parameters fluctuating independently and simultaneously, the collective effect of multi-parameters can be straightly obtained. For a typical InGaAsP/InP singlephoton avalanche diode, it is seen that device homogeneity with excess bias fluctuation within $50 \%$ requires uncertainty in layer thickness and doping level better than $\sim 3 \%$.
\end{abstract}

\section{Introduction}

Single photon avalanche diodes (SPADs) [1] are widely investigated and developed for applications in many fields such as single photon imaging and quantum information processing, due to their advantageous features like small size, low bias, low power consumption and reliability, which are superior to other single photon detectors such as photomultiplier tubes and superconducting single photon detectors. InP-based Geiger-mode avalanche photodiode (APD) is an excellent SPAD for the near-infrared wavelength region (1.0-1.7 $\mu \mathrm{m})$, especially as a focal plane array detector. Owing to the advanced epitaxial technology, InP-based SPADs are well developed and have been applied in recent years. However, there are still many critical problems to be resolved, one of which is the reproducibility and/or uniformity [2], which should be well-controlled by controlling the parameters in epitaxy and device process. Although one can estimate the influence of some individual parameters on the reproducibility/uniformity by an analytical method [3], it is difficult to clarify the collective effect from multiple parameters and impossible to quantitatively make tradeoff between various parameters. It is necessary to establish the quantitative correlation between the inhomogeneity in device characters and the fluctuation in all the epitaxy/process parameters. In this work, we perform a statistical analysis on device property by introducing random fluctuations in many structure parameters, and we give the control precisions of a few parameters required for good device reproducibility/uniformity for typical InGaAsP/InP SPADs.

\section{Method}

We design an InGaAsP/InP APD structure as an exampling object. As shown schematically in Fig.1(a), it has a hetero-structure consisting of separated absorption, charge, and multiplication layers. The InGaAsP absorption layer is designed to have band gap of $1.2 \mu \mathrm{m}$ so as to get a peak response at $1.06 \mu \mathrm{m}$. Fig. 1(b) and (c) show the device characteristics of this structure, which are calculated using conventional APD theory [4] and recently advanced methods [5]. The simulated results of $I-V$ characteristics without illumination is shown in Fig. 1(b), presenting individually the tunneling current, the generation/recombination current, and the total dark current with/without avalanche at $230 \mathrm{~K}$. The breakdown voltage $V_{\mathrm{b}}$ is defined as the value of applied reverse bias $V$ corresponding to infinite avalanche current. The Geige-mode $\left(V>V_{\mathrm{b}}\right)$ dark current without 
avalanche at $300 \mathrm{~K}$ are contributed by both the generation/recombination and tunneling currents (not shown), while that at $230 \mathrm{~K}$ are almost solely determined by the tunneling current. Figure 1(c) shows the dark count rate (DCR), the rate that an avalanche count occurs without any photon arrival, versus photon detection efficiency (PDE), the probability to record a count when there are incident photons (wavelength of $1.06 \mu \mathrm{m}$ herewith). They are two most fundamental performance indices for a Geige-mode APD. Depending on the applied excess bias $V_{\mathrm{ex}}=V-V_{\mathrm{b}}$, DCR is getting worse while PDE is getting better. Operating at a middle excess bias $V_{\mathrm{ex} 0}=5 \mathrm{~V}$, which will be taken as the reference point in the following simulation, is the optimal choice.

Taking the above structure parameters and the fundamental properties as a reference, we try studying the variation of device characters as a result of parameter fluctuations. In the application of SPAD arrays, thousands of pixels are generally biased by a common voltage $V_{0}$. If the individual pixels have structure fluctuation, the effective excess bias will be different from each other and then there occur inhomogeneity of device performance such as DCR and PDE. To study this effect, we randomly change the device structures in a way as, for any structure parameter $t$, allowed.

$$
t_{i}=t_{0}\left(1+W \sigma_{\mathrm{i}}\right)
$$

where $\mathrm{i}=1,2,3 \ldots$ denotes the cycle number of calculation trial, $t_{0}$ is the designed value of $t$ as presented in Fig.1(a), $W$ is an arbitrary constant determining the distribution width and $\sigma_{\mathrm{i}}$ is a series of random values normally distributed with full width at half maximum (FWHM) of 1 . In this work,

(a)

\begin{tabular}{|c|}
\hline $\begin{array}{l}\mathrm{p}^{+} \text {-InP, } 3 \mu \mathrm{m} \\
\text { Zn-doping } 5 \times 10^{18} \mathrm{~cm}^{-3}\end{array}$ \\
\hline $\begin{array}{l}\text { multiplication layer } \\
\text { InP } 1.5 \mu \mathrm{m} \\
\text { residual doping } 1 \times 10^{15} \mathrm{~cm}^{-3}\end{array}$ \\
\hline $\begin{array}{l}\text { charge layer, InP, } 0.2 \mu \mathrm{m} \\
\text { Si-doping } 1 \times 10^{17} \mathrm{~cm}^{-3}\end{array}$ \\
\hline $\begin{array}{l}\text { absorption layer } \\
\text { InGaAsP } 2 \mu \mathrm{m} \\
\text { residual doping } 1 \times 10^{15} \mathrm{~cm}^{-3}\end{array}$ \\
\hline $\begin{array}{l}\mathrm{n}-\ln \mathrm{P}, 1 \mu \mathrm{m} \\
\text { Si-doping } 5 \times 10^{17} \mathrm{~cm}^{-3}\end{array}$ \\
\hline $\begin{array}{l}\mathrm{n}^{+}-\mathrm{InP} \text { substrate } \\
350 \mu \mathrm{m}\end{array}$ \\
\hline
\end{tabular}
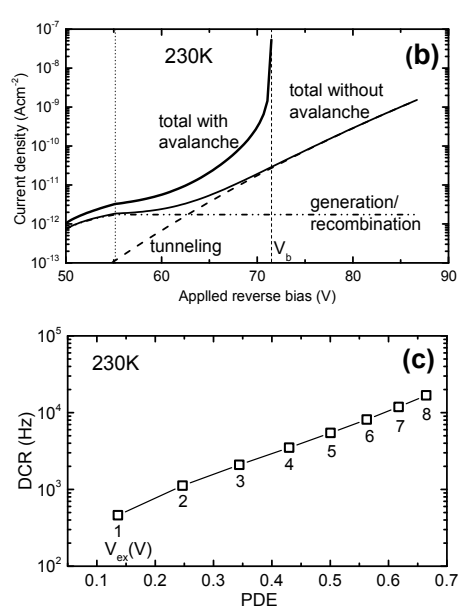

Fig.1. (a) The epitaxial structure of a typical InGaAsP /InP Geige-mode APD used in this study; simulated (b) $I-V$ characteristics and (c) DCR versus PDE of that device. Since InP-based APD devices normally operate under cooled condition, we mainly focus on the device properties at $230 \mathrm{~K}$.

\section{Results and Discussions}

The simplest case is to study the fluctuation effect of a single parameter. By giving 1000 randomly varying values of absorption width ta normally distributed with FWHM of $3 \%\left(W_{\mathrm{ta}}=3 \%\right)$ while remaining other structure parameters invariable, we obtain the fluctuation distribution of excess bias $V_{\text {ex }}$ as shown in Fig. 2(a). We see that the resulted $V_{\text {ex }}$ fluctuation also exhibits a normal distribution, i.e. it can also be expressed by Eq. 1. Its distribution FWHM is $4.8 \%$. By the same way, a $3 \%$ fluctuation in multiplication width $t_{\mathrm{m}}$ leads to a normal $V_{\text {ex }}$ distribution with FWHM of $20 \%$, as shown by Fig. 2(b). It implies that different parameters fluctuate the device properties to quite different degrees. More detailed and systematic simulations make clear how significant any 
parameter is for a Gm-APD device. Figure 2(c) shows the $V_{\text {ex }}$ distribution width as functions of the fluctuations of single parameters including the width/doping of the multiplication region $t_{\mathrm{m}} / n_{\mathrm{m}}$, absorption region $t_{\mathrm{a}} / n_{\mathrm{a}}$, and charge region $t_{\mathrm{c}} / n_{\mathrm{c}}$. We see that three parameters $t_{\mathrm{m}}, t_{\mathrm{c}}$ and $n_{\mathrm{c}}$, have strong effects, whereas other three $n_{\mathrm{a}}, t_{\mathrm{a}}, n_{\mathrm{m}}$ have weaker effects on excess bias $V_{\text {ex }}$. The strongest case is the doping concentration in the charge layer $n_{\mathrm{c}}$. Fluctuation of only $3 \%$ in $n_{\mathrm{c}}$ may cause $V_{\mathrm{ex}}$ fluctuation of more than $30 \%$. It suggests that the charge quantity should be considered most carefully in design and controlled most precisely in epitaxy process. The weakest case is the doping concentration in multiplication layer $n_{\mathrm{m}}$. Fluctuation of $10 \%$ in $n_{\mathrm{m}}$ only brings about $5 \%$ fluctuation in $V_{\text {ex }}$. Besides, every parameter linearly generate $V_{\text {ex }}$ fluctuation. As a reference, let us mark the parameter fluctuation FWHM leading to $10 \%$ of $V_{\mathrm{ex}}$ fluctuation as $W_{-10}$. From the data in the Fig. 2(c), we learn that the $W_{-10}$ values of the absorption-region doping and width, the multiplicationregion doping and width, and the charge-region doping and width are $W_{\text {na- } 10}=19 \%, W_{\text {ta- } 10}=6.5 \%$, $W_{\mathrm{nm}-10}=23 \%, W_{\mathrm{tm}-10}=1.5 \%, W_{\mathrm{nc}-10}=0.5 \%$, and $W_{\mathrm{tc}-10}=0.9 \%$, respectively.

$V_{\text {ex }}$ deviation (\%)

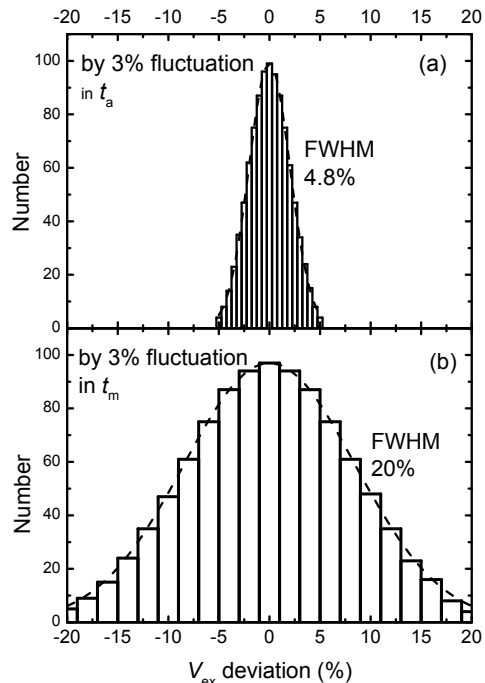

It is more important to know several fluctuated parameters influence the precision of device properties. Firstly, let us consider the case of 2 parameters. Among the above six parameters, we choose arbitrarily two, e.g. $\left(t_{\mathrm{m}}, t_{\mathrm{a}}\right)$. As a typical condition, we take $10 \%$ of $V_{\mathrm{ex}}$ fluctuation in the case of single varying parameters, corresponding to $W_{\mathrm{tm}-10}=1.5 \%$ of $t_{\mathrm{m}}$ fluctuation or $W_{\mathrm{ta}-}$ ${ }_{10}=6.5 \%$ of $t_{\mathrm{a}}$ fluctuation as can be deduced from Fig 2(b), as a reference. In detail, we set $t_{\mathrm{mi}}=t_{\mathrm{m} 0}\left(1+W_{\mathrm{tm}}\right.$ $\left.{ }_{10} \sigma_{\mathrm{tmi}}\right)$ and $t_{\mathrm{ai}}=t_{\mathrm{a} 0}\left(1+W_{\mathrm{ta}-10} \sigma_{\mathrm{tai}}\right)$ and keep other parameters invariable. Thousands of $\left(\sigma_{\text {tmi }}, \sigma_{\text {tai }}\right)$ thus generate thousands of device character data sets $\left(V_{\text {exi }}, \mathrm{DCR}_{\mathrm{i}}, \mathrm{PDE}_{\mathrm{i}}\right)$. Statistics over these results give the effect of

Fig. 2. Distribution of excess bias $V_{\text {ex }}$ induced by a $3 \%$ normally fluctuated single parameter (a) absorption region width $t_{\mathrm{a}}$, (b) multiplication-region width $t_{\mathrm{m}}$; (c) $V_{\text {ex }}$ fluctuation FWHM varying when a single parameter is changing.

simultaneously fluctuating $t_{\mathrm{m}}$ and $t_{\mathrm{a}}$. The resulted $V_{\text {ex }}$ fluctuation FWHM is $15 \%$, less than a simple summation of single parameter effects $20 \%$. Similarly changing other two arbitrary parameters, which have 14 choices such as $\left(t_{\mathrm{m}}, t_{\mathrm{c}}\right),\left(t_{\mathrm{c}}, n_{\mathrm{c}}\right),\left(n_{\mathrm{c}}, n_{\mathrm{a}}\right)$ and so on, we get sets of device property data. Statistics on these data sets implies that the $V_{\text {ex }}$ fluctuation generated by two parameters, normally and independently fluctuating with FWHM of $W_{-10}$, is $(15 \pm 2) \%$. Extending the above process to more parameters, we obtain the fluctuation of device property as a function of parameter numbers. The result for $V_{\mathrm{ex}}$ is shown in Fig. 3(a), where we see a sublinear change of $V_{\text {ex }}$ fluctuation with increasing parameter number. As all of the six parameters are taken into accounted, the excess bias $V_{\text {ex }}$ fluctuates with FWHM of $24 \%$, far below a simple summation over single parameter effects. It suggests that the
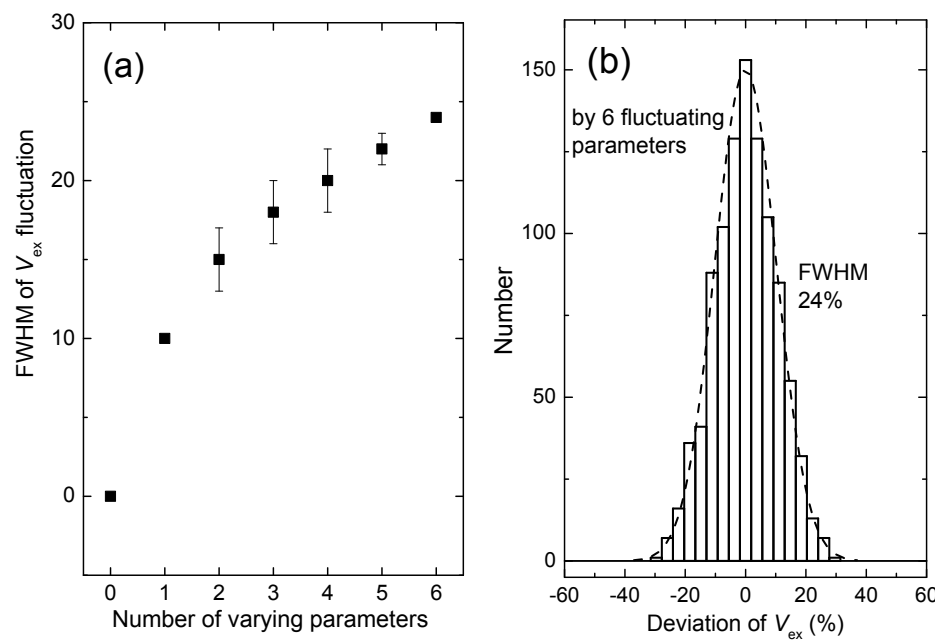

Fig.3. (a) Fluctuation of the excess bias $V_{\text {ex }}$ as a function of the number of fluctuating parameters; (b) $V_{\text {ex }}$ distribution caused by 6 fluctuating parameters 
required limits in epitaxial condition may be greatly relaxed with respect to intuitive estimates. The $V_{\text {ex }}$ distribution caused by six independently fluctuating parameters is shown in Fig. 3(b). Since the referred excess bias $V_{\text {ex } 0}=5 \mathrm{~V}$, the practical excess bias varies mainly within 4.4-5.6V, which looks tolerable in application. It is also important to know the effect on other device characters.

What is more meaningful, we shall see how the effect is if all the parameter fluctuations change. We check firstly a case where all parameters fluctuate with FWHM $\beta$ times the above value $W_{-10}$. That is to set $n_{\mathrm{ai}}=n_{\mathrm{a} 0}\left(1+\beta W_{\mathrm{na}-10} \sigma_{\mathrm{nai}}\right), t_{\mathrm{ai}}=t_{\mathrm{a} 0}\left(1+\beta W_{\mathrm{ta}-10} \sigma_{\mathrm{tai}}\right), n_{\mathrm{mi}}=n_{\mathrm{m} 0}\left(1+\beta W_{\mathrm{nm}-10} \sigma_{\mathrm{nmi}}\right), t_{\mathrm{mi}}=$ $t_{\mathrm{m} 0}\left(1+\beta W_{\mathrm{tm}-10} \sigma_{\mathrm{tmi}}\right), n_{\mathrm{ci}}=n_{\mathrm{c} 0}\left(1+\beta W_{\mathrm{nc}-10} \sigma_{\mathrm{nci}}\right)$, and $t_{\mathrm{ci}}=t_{\mathrm{c} 0}\left(1+\beta W_{\mathrm{tc}-10} \sigma_{\mathrm{tci}}\right)$. The result is presented in Fig. 4 by circles. It indicates that $V_{\text {ex }}$ fluctuation increases proportionally with parameter fluctuation times $\beta$. When $\beta=2, V_{\text {ex }}$ varies normally with FWHM of nearly $50 \%$, which means $V_{\text {ex }} \sim 3.8-6.2 \mathrm{~V}$, acceptable in application. In this case, the fluctuations of $n_{\mathrm{a}}, t_{\mathrm{a}}, n_{\mathrm{m}}$ are respectively $38 \%, 13 \%$ and $46 \%$, easy to realize in epitaxy; while those of $t_{\mathrm{m}}, n_{\mathrm{c}}$, and $t_{\mathrm{c}}$ should be better than $3 \%, 1.8 \%$ and $2 \%$

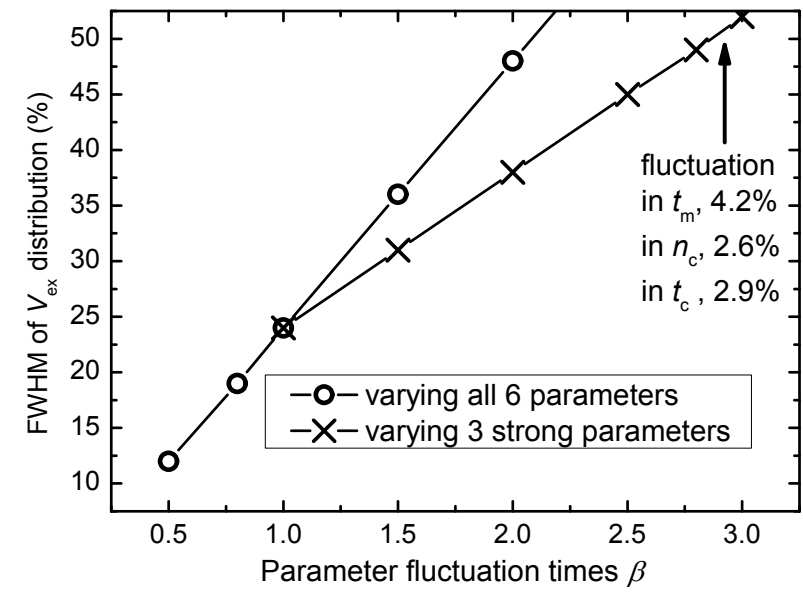

Fig. 4. $V_{\text {ex }}$ fluctuation varying as all the six parameters or only 3 parameters $t_{\mathrm{m}}, n_{\mathrm{c}}, t_{\mathrm{c}}$ increase their fluctuations. respectively, challenging for epitaxy growth. There may be some way to relax these strict requirements. Let us keep $n_{\mathrm{ai}}=n_{\mathrm{a} 0}\left(1+W_{\text {na- } 10} \sigma_{\text {nai }}\right)$, $t_{\mathrm{ai}}=t_{\mathrm{a} 0}\left(1+W_{\mathrm{ta}-10} \sigma_{\mathrm{tai}}\right), n_{\mathrm{mi}}=n_{\mathrm{m} 0}\left(1+W_{\mathrm{nm}-10} \sigma_{\mathrm{nmi}}\right)$, i.e. remaining the fluctuations of weaker parameters at the reference level $W_{-10}$, while setting other parameters $t_{\mathrm{m}}, n_{\mathrm{c}}$, and $t_{\mathrm{c}}$ as the above, i.e. varying the stronger parameters with FWHM of $\beta W_{-10}$. The result shown by crosses in Fig. 4 indicates also a linear increase of $V_{\mathrm{ex}}$ fluctuation with $\beta$. More importantly, a $V_{\text {ex }}$ fluctuation of $\sim 50 \%$ corresponds to $\beta \sim 2.9$, meaning that $t_{\mathrm{m}}, n_{\mathrm{c}}$, and $t_{\mathrm{c}}$ can vary within $4.2 \%, 2.6 \%$ and $2.9 \%$ respectively. A tradeoff among multiple parameters is thus practical for the design of SPAD arrays. If we set weaker parameters fluctuating less than $W_{-01}$, there will be more relaxation space in stronger parameters.

\section{Summary}

For Geiger-mode APDs, we propose a method to quantitatively correlate the controllability of structure parameters with the homogeneity of device properties. By setting many parameters fluctuating independently and simultaneously, the collective effect of multi-parameters can be obtained and a design tradeoff between different parameters becomes straightforward.

\section{References}

[1] G. Boso, B. Korzh, T. Lunghi and H. Zbinden, Low noise InGaAs/InP single-photon negative feedback avalanche diodes: characterization and applications, Proc. SPIE 9492 (2015) 94920Q.

[2] M. A. Itzler, U. Krishnamachari, Q. Chau, X. D. Jiang, M. Entwistle, M. Owens, and K. Slomkowski, Statistical analysis of dark count rate in Geiger-mode APD FPAs, Proc. SPIE 9250 (2014) 925003.

[3] V. Savuskan, I. Brouk, M. Javitt, and Y. Nemirovsky, An Estimation of Single Photon Avalanche Diode (SPAD) Photon Detection Efficiency (PDE) Nonuniformity, IEEE Sensors J. 13 (2013) 1637-1640.

[4] W. T. Tsang (translated by Baoxun Du), Semiconductor optical detectors, Beijing, 1992.

[5] J. P. Donnelly, E. K. Duerr, K. Alex McIntosh, E. A. Dauler, D. C. Oakley, S. H. Groves, C. J. Vineis, L. J. Mahoney, K. M. Molvar, P. I. Hopman, K. E. Jensen, G. M. Smith, S. Verghese, and D. C. Shaver, IEEE J. Quant. Electr. 42 (2006) 797-809. 